\title{
Solubility of acyclovir in nontoxic and biodegradable ionic liquids: COSMO-RS prediction and experimental verification
}

\begin{abstract}
The pharmaceutical industry faces a challenge to find potential solvents for drug molecules that are sparingly soluble in water and organic solvents. Recently, ionic liquids (ILs) have attracted great attention as pharmaceutical solvents owing to their unique physicochemical and biological properties. In this study, the solubility of the sparingly soluble drug molecule acyclovir (ACV) in a wide variety of ILs was investigated by conductor-like screening model for real solvents (COSMO-RS) calculations. The predicted solubilities were validated by experimental measurements, and good agreement was found between the predicted and experimental results. The solubility of ACV was greatly affected by the structure of the ILs, particularly the anionic moiety. Among the various ILs tested, ACV showed excellent solubility in ammonium-based ILs with an acetate anion. In vitro cytotoxicity of ILs to the MCF-10 normal breast epithelial cell line and cancer cell lines (MDA MB 231 and MCF 7) was investigated. The ammonium-based ILs showed higher IC50 values than the imidazolium-based ILs with the acetate anion. Biodegradability results showed that diethylammonium acetate, triethylammonium acetate, and choline acetate ILs have high levels of biodegradation under aerobic conditions and can be classified as readily biodegradable. These findings will be useful for the design of IL-based drug delivery carriers that can act as versatile and efficient drug delivery systems for sparingly soluble drug molecules.
\end{abstract}

Keyword: Acyclovir; Ionic liquid; Solubility; COSMO-RS; Biodegradability; Cytotoxicity 
